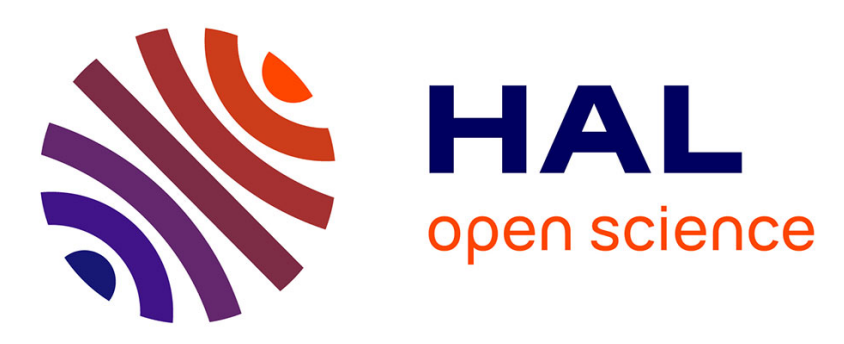

\title{
Phased and synchronous sampling between multiple smart network sensors for modal assessment of large structures
}

Mathieu Le Pen, Arthur Bouché, Ivan Guéguen, Michael Döhler, Laurent

Mevel, Vincent Le Cam

\section{To cite this version:}

Mathieu Le Pen, Arthur Bouché, Ivan Guéguen, Michael Döhler, Laurent Mevel, et al.. Phased and synchronous sampling between multiple smart network sensors for modal assessment of large structures. IWSHM 2019 - 12th International Workshop on Structural Health Monitoring, Sep 2019, Stanford, CA, United States. pp.1-9, 10.12783/shm2019/32228 . hal-02290901

\section{HAL Id: hal-02290901 \\ https://hal.inria.fr/hal-02290901}

Submitted on 18 Sep 2019

HAL is a multi-disciplinary open access archive for the deposit and dissemination of scientific research documents, whether they are published or not. The documents may come from teaching and research institutions in France or abroad, or from public or private research centers.
L'archive ouverte pluridisciplinaire HAL, est destinée au dépôt et à la diffusion de documents scientifiques de niveau recherche, publiés ou non, émanant des établissements d'enseignement et de recherche français ou étrangers, des laboratoires publics ou privés. 
Title: Phased and synchronous sampling between multiple smart network sensors for modal assessment of large structures

\author{
Authors : Mathieu Le Pen \\ Arthur Bouche \\ Ivan Guéguen \\ Michael Döhler \\ Laurent Mevel \\ Vincent Le Cam
}




\begin{abstract}
Operational modal analysis and vibration-based damage detection of engineering structures have become important issues for Structural Health Monitoring (SHM) and maintenance operations. For this task, embedded wireless platforms such as the PEGASE platform are appealing and suitable to collect vibration data and then perform off-line and remote computation easily. To obtain detailed modal information of large and very large structures, many sensors would be required to cover the geometry of the structure with a reasonable accuracy. However, when only a limited amount of sensors is available, large structures can be measured in several sensor setups, where some sensors remain fixed and some are moved between different measurement setups. With the sensors connected to different wireless platforms, the synchronous acquisition of data is required. In this paper, a solution of data acquisition synchronization, as well as signal processing for merging the information taking into account the change of sensor positions and environmental variability is presented.
\end{abstract}

\title{
INTRODUCTION
}

Operational modal analysis and vibration-based damage detection of engineering structures have become important issues for Structural Health Monitoring (SHM) and maintenance operations [1]. In order to retrieve information about the structural health, modal analysis is often a first step to be carried out, retrieving the modes (natural frequencies, damping ratios and mode shapes) of the structure from ambient vibration measurements. To obtain detailed modal information of large and very large structures, many sensors would be required to cover the geometry with a reasonable accuracy. This kind of instrumentation is beyond the reach of most experimental works. For this purpose, and many others, wireless platforms such as the PEGASE platform [2] are a good candidate for managing such a large-scale instrumentation. With the practical difficulties to access structures, to collect data and then perform off-line and remote computation, embedded wireless sensor networks (WSN) indeed offer an advantage compared to clas-

M. Le Pen, I. Guéguen, V. Le Cam, Ifsttar COSYS/SII, Inria, Bouguenais, France.

A. Bouche, Ifsttar COSYS/SII, Bouguenais, France.

M. Döhler, L. Mevel, Univ. Rennes, Inria, Ifsttar COSYS/SII, Rennes, France. 
sical measurement systems [3].

A rather straightforward setup would be to manage a large amount of sensors using one or many wireless platforms communicating all together. However, the resulting costs in sensors and hardware of this approach may not be reasonable. Another solution is the use of a limited amount of sensors, of which some are fixed and some are moved between different measurement setups to obtain vibration measurements at many coordinates of the structure with only few sensors $[4,5]$. This corresponds to managing one or several moving wireless platforms and record data on the structure in multiple setups. However, this cheaper alternative requires perfect synchronization of data time stamps and perfect phasing acquisition between different platforms. In particular, a solution of data acquisition synchronization, as well as signal processing for merging the information taking into account the change of sensor positions and environmental variability are required. This is addressed in this paper. The approach is tested on relevant numerical and laboratory experimental data.

\section{SYNCHRONOUS MEASUREMENTS WITH PEGASE PLATFORMS}

PEGASE is the commercial name of a generic Wireless Sensor Platform conceived and designed by IFSTTAR since 2008. The PEGASE concept is essentially based on a generic vision of its hardware and software abilities. Hardware genericity is provided by a principle of mother and pluggable daughter boards.

The PEGASE mother board (Figure 1a) integrates most common functions of typical wireless systems: ensure computation, manage energy, offer multiple I/Os and wireless communications. One of the outstanding capabilities of the PEGASE mother board is the accurate absolute time synchronization up to 50 nanoseconds UT based on a GPS/PPS receiver and real-time algorithm in a driver [6].

Each pluggable daughter board adds a specific function to the mother board, such as an eight channels ADC daughter board (Figure 1b), allowing eight analog and eight digital inputs. Amongst its properties are a differential analog inputs range of \pm 2.5 Volt, 24 bits of analog/digital synchronous converter channels and up to $34 \mathrm{kHz}$ of sampling frequency.

Software genericity is provided through a Linux Operating System added to a Single

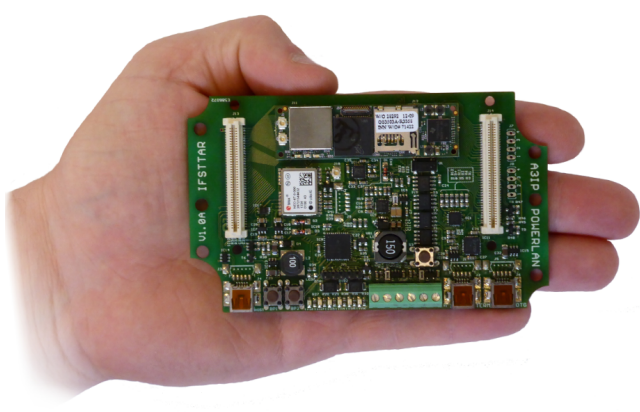

(a) The PEGASE mother board

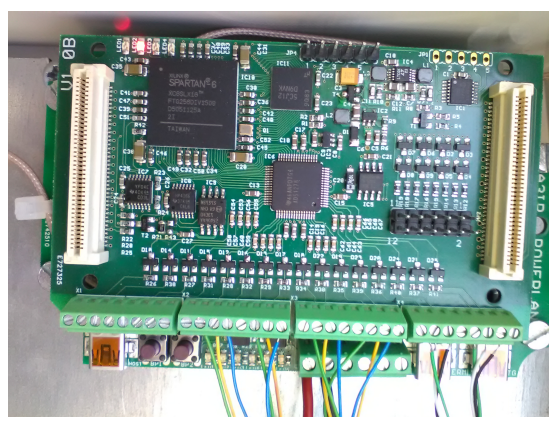

(b) ADC 8 channels daughter board

Figure 1: PEGASE concept 
Development Kit (SDK) given in C++ open-source in object-oriented languages.

\section{Problem statement}

Some applications, such as vibration measurements for multiple sensor setups, require phasing and synchronous acquisition of data from several PEGASE sensor platforms. In this application, some sensors are fixed on a structure, while other sensors are moved between different measurement setups, which allows to record vibration data at many locations of a structure with a limited amount of sensors. For example, the fixed sensors may be connected to one PEGASE platform, while the moving sensors may be connected to another platform or several other platforms, depending on the number of available sensors. The measurements with the different platforms need to be perfectly phased to allow the subsequent treatment of the measured data for modal analysis.

\section{Synchronization and phasing of measurements}

At the current point of development, the driver of the daughter board does not yet integrate the Frame Synchronization mode allowing to clock the data acquisition. Because of its mode of control (SPI mode), the start time of the acquisition of the ADC and the sampling frequency cannot be determined in advance. It is thus impossible to guarantee an acquisition of data samples in phase and simultaneous between two different ADC. In order to ensure measurements that are nonetheless in phase, we have developed and qualified a method for reconstructing samples by linear interpolation on a common time basis between different embedded PEGASE platforms.

For this purpose, we have developed an algorithm that distributes a timestamp tOrigin between each platform via a local area network (LAN). PEGASE wireless synchronization technology ensures that all PEGASE sensors share the same time base with an error of less than 50 nanoseconds. A master sensor initiates a TCP server and the TCP clients of slave sensors periodically try to connect to this server. All sensors launch their acquisitions to continuously measure vibration data and save them periodically in a circular buffer. Due to the minimum sampling rate applicable to the ADC $(99 \mathrm{~Hz})$ and in order to minimize the interpolation error, over sampling is applied with a factor 10 of the desired sampling frequency.

When a measurement is requested (at the push of a button by the operator), the timestamp tOrigin of this event is sent to the master server which saves it and passes it back to all connected slave clients. Upon receipt of a timestamp tOrigin, the board will search the start tOrigin and end time $t$ End of the requested setup in the circular buffer, scan the circular buffer to verify that the requested time frame is fully present (i.e. between the sample before tOrigin and the sample after $t$ End), and extract a copy to be processed. The resulting data is usually not synchronous between different boards, as illustrated in Figure 2. For each timestamp tOrigin received, each board reconstructs a data set by interpolation between each pair of measurement points for each connected sensor, which is synchronous between the boards, by

$$
x(\text { tOrigin }+k / f \text { Wanted })=x\left(t^{\prime}\right)+\frac{x\left(t^{\prime \prime}\right)-x\left(t^{\prime}\right)}{t^{\prime \prime}-t^{\prime}}\left(k / f \text { Wanted }-t^{\prime}\right)
$$

where $k$ is the number of the sample, fWanted the desired sampling frequency, and $t^{\prime}$ 


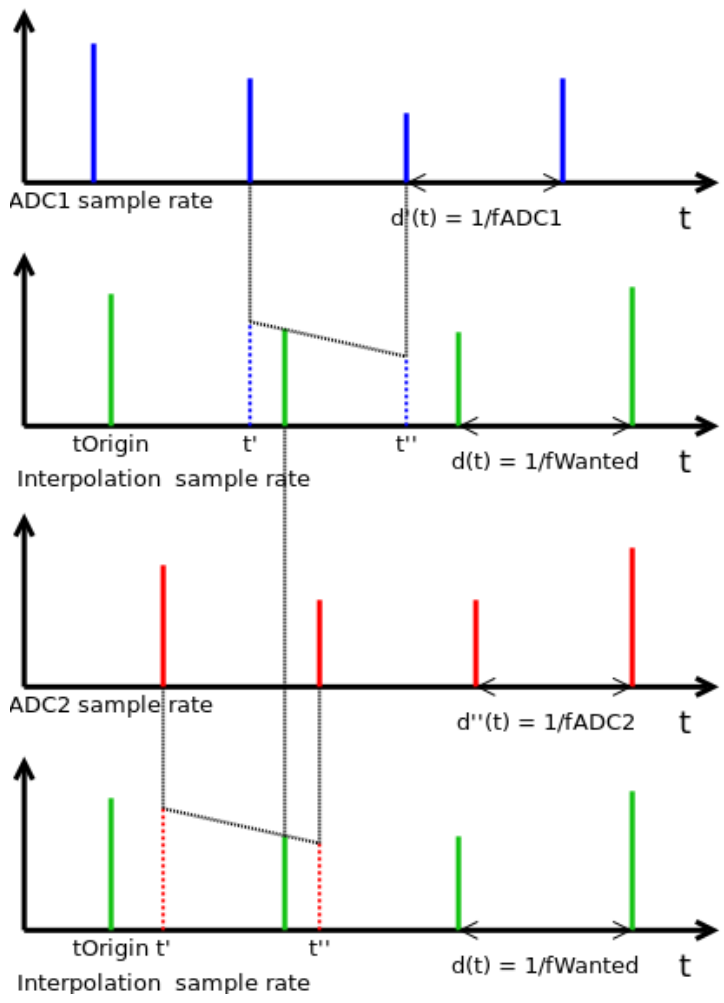

Figure 2: ADC unphasing and interpolation restitution

and $t^{\prime \prime}$ are the time indices before and after the desired time instant for sample reconstruction with $t^{\prime} \leq$ tOrigin $+k / f$ Wanted $\leq t^{\prime \prime}$.

Then we applied an anti-aliasing LPF filter and decimated the signal by factor 10 to obtain a data configuration starting at time tOrigin and with in-phase data samples. This process is parallelized so that several successive setups can be requested. Valid setups are finally returned to the master board that controls the correct reception of all the setups requested from each client before assembling them for the application of further signal processing.

\section{MODAL ANALYSIS FROM MULTIPLE SETUPS}

To obtain vibration measurements at many coordinates of a structure with only few sensors, it is common practice to use multiple sensor setups for the measurements. For these multi-setup measurements, some of the sensors, the so-called reference sensors, stay fixed throughout all the setups, while the other sensors are moved from setup to setup. By merging in some way the corresponding data while taking into account possible different ambient excitation between the measurements, this allows to perform modal identification as if there was a large number of sensors. Based on subspace identification $[7,8]$, a global merging approach was proposed in $[4,5]$ that is recalled here briefly.

In each measurement setup $j$, data $y_{k}^{(j, \text { ref })}$ of dimension $r^{(\text {ref })}$ is recorded from a fixed reference sensor pool, and data $y_{k}^{(j, \text { mov })}$ of dimension $r^{(j)}$ from a moving sensor pool. To 


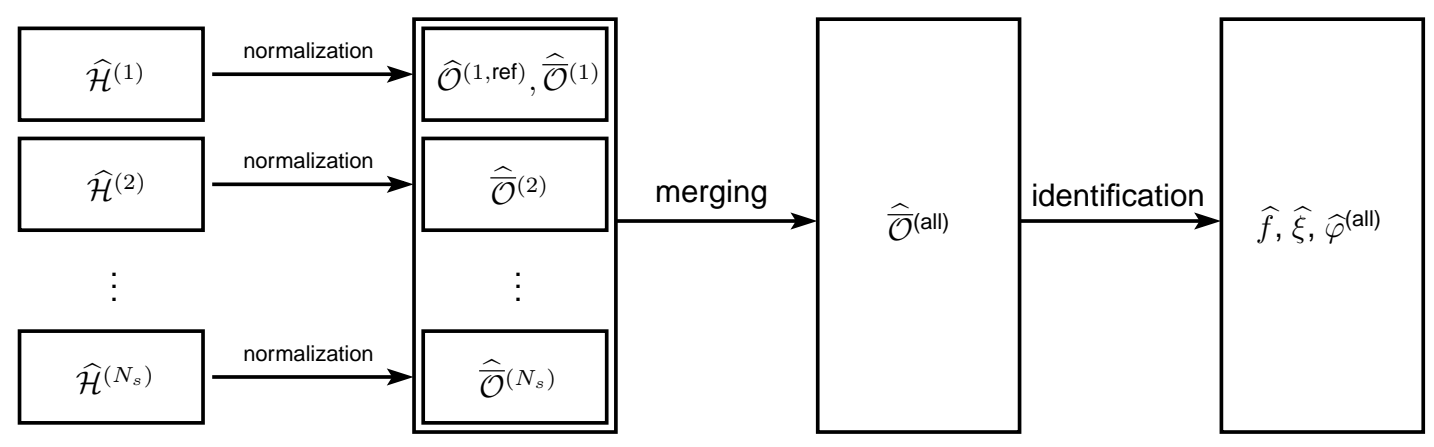

Figure 3: Merging and system identification scheme for modal analysis of multiple sensor setups.

each record $j=1, \ldots, N_{s}$ corresponds a state-space realization in the form [4]

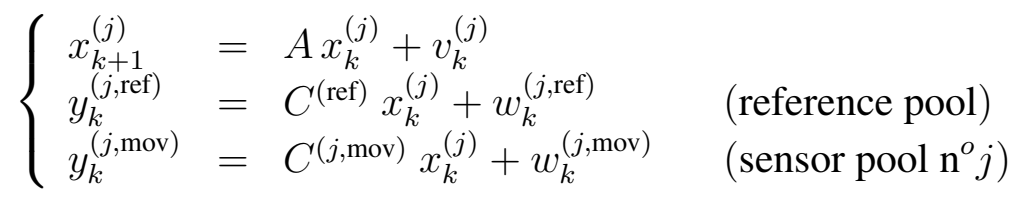

with a single state transition matrix $A$, since the same system is being observed, while the output matrices $C^{(\text {ref })}$ and $C^{(j, \mathrm{mov})}$ depend on the sensor positions of the reference sensors and the moving sensors for each measurement setup, respectively. With the algorithm described in $[4,5]$, the global observability matrix $\overline{\mathcal{O}}^{(\text {all })}$ of the system is obtained with

$$
\overline{\mathcal{O}}^{(\text {all })}=\left[\begin{array}{c}
C \\
C A \\
\vdots \\
C A^{p}
\end{array}\right], \quad \text { where } C=\left[\begin{array}{c}
C^{(\text {ref })} \\
C^{(1, \mathrm{mov})} \\
\vdots \\
C^{\left(N_{s}, \mathrm{mov}\right)}
\end{array}\right]
$$

containing the information of all sensor positions. This is done by extracting the observability matrix part in each setup and normalizing it with respect to the reference sensor part of a chosen setup. Then, the global system matrices $A$ and $C$ are extracted as usual in subspace identification from $\overline{\mathcal{O}}^{\text {(all) }}$, and subsequently the modal parameters are obtained. A scheme of the approach is depicted in Figure 3.

\section{TEST ON A REAL STRUCTURE}

After validating the synchronization and interpolation algorithm on two PEGASE platforms with an artificial electronic signal, where a discrepancy in the recorded and interpolated signals of $0.11 \%$ has been found, a vibration test on a beam structure was carried out (Figure 4).

The measurement chain consisted of Silicon Designs 2210-002 1-axis accelerometers $(2000 \mathrm{mV} / \mathrm{g})$ and a Texas Instruments ADS 1278 analog / digital converter (24 bits \pm 2.5 Vref $)$. The total measurement precision was 5 milli g. The accelerometers were arranged on the top of the beam, oriented in $Z$ direction, in the configuration as shown in Figure 5. Data was recorded during 1 min for each test, with the goal of obtaining 


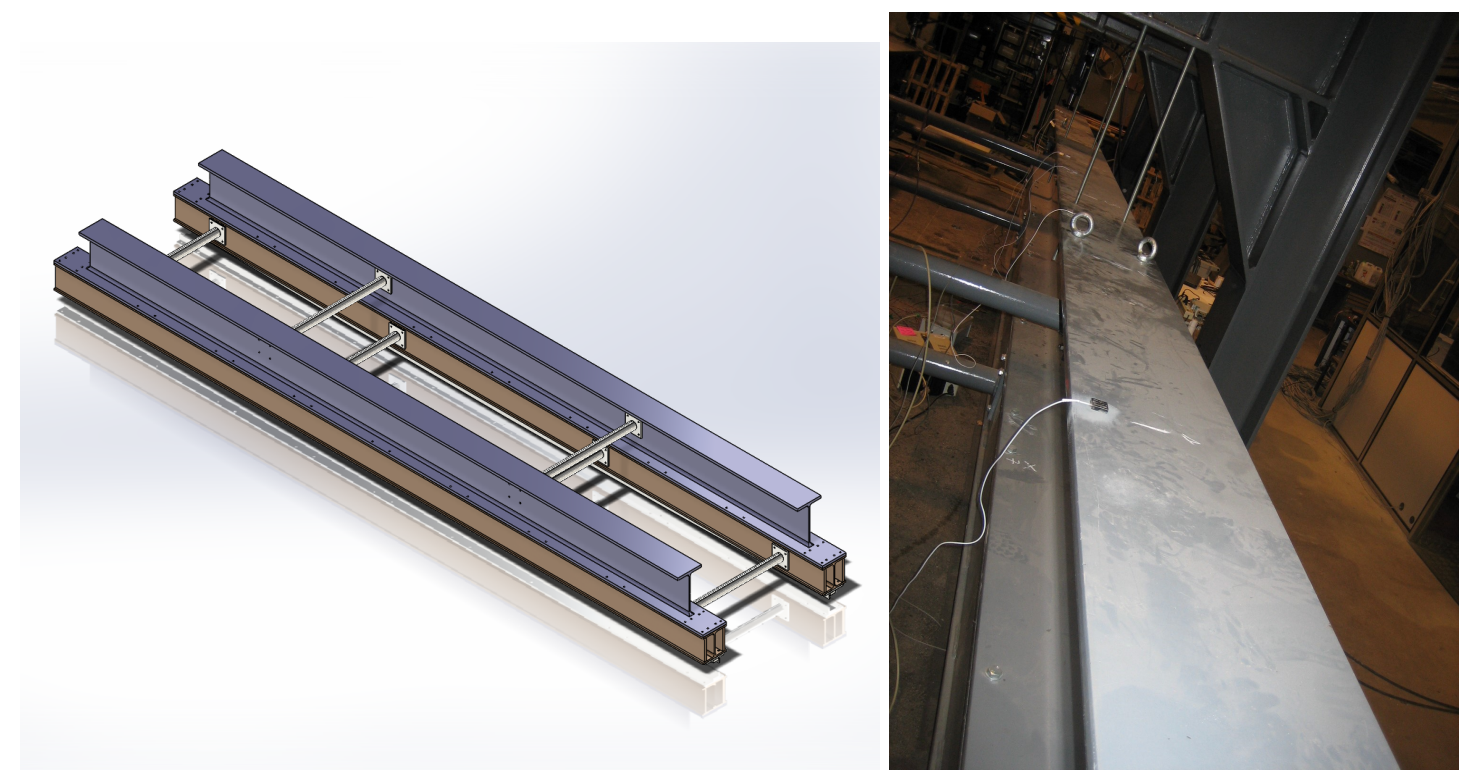

Figure 4: Test beam with instrumentation

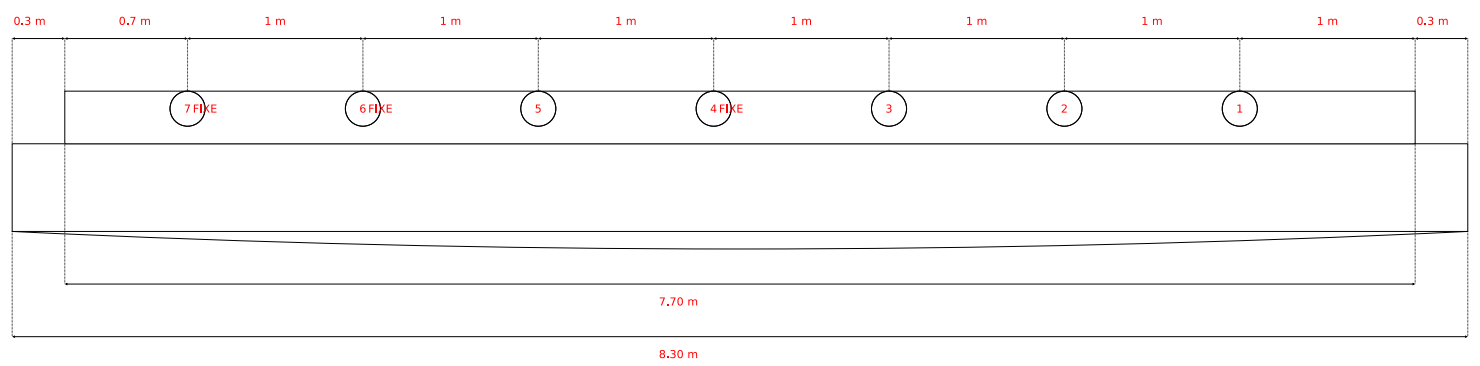

Figure 5: Placement of sensors on beam

12000 synchronous samples at a sampling rate of $200 \mathrm{~Hz}$. For each test, the sensors 4, 6 and 7 remained fixed and were connected to the PEGASE master board, while sensors 1, 2, 3 and 5 may be moved. The following tests were carried out:

- Test 1: sensors 1, 2, 3 and 5 were also connected to the PEGASE master board. Here, one measurement setup was recorded with one card.

- Test 2: sensors 1, 2, 3 and 5 were connected to the PEGASE slave board. One measurement setup was recorded with two cards.

- Test 3: a first setup was carried out by activating the sensors 1 and 2 connected to the PEGASE slave board, and a second setup by activating the sensors 3 and 5 connected to the PEGASE slave board. Here, two measurement setups were recorded with the two cards, corresponding to the multi-setup configuration.

From the data collected in this three tests, modal analysis was performed with the covariance-driven subspace-based system identification, either directly for Tests 1 and 2, or using the multi-setup algorithm from the previous section for Test 3 . The identification was carried out using $p=100$ time lags and using the three reference sensors in all tests. In Figure 6, the stabilization diagram is shown with Test 1, where several modes can 
be identified. Five modes were selected for comparison. The identified frequencies and damping ratios are shown in Table I, and the mode shapes in Figure 7 for the three tests. In general, a very good agreement between the identified modal parameters using one card (without synchronization) or several cards (with synchronization) can be found, validating the proposed strategy for synchronous data recording and processing. Note that some differences appear in the damping ratio of mode 1 between the different tests, which is the most difficult to identify and therefore prone to more uncertainty.

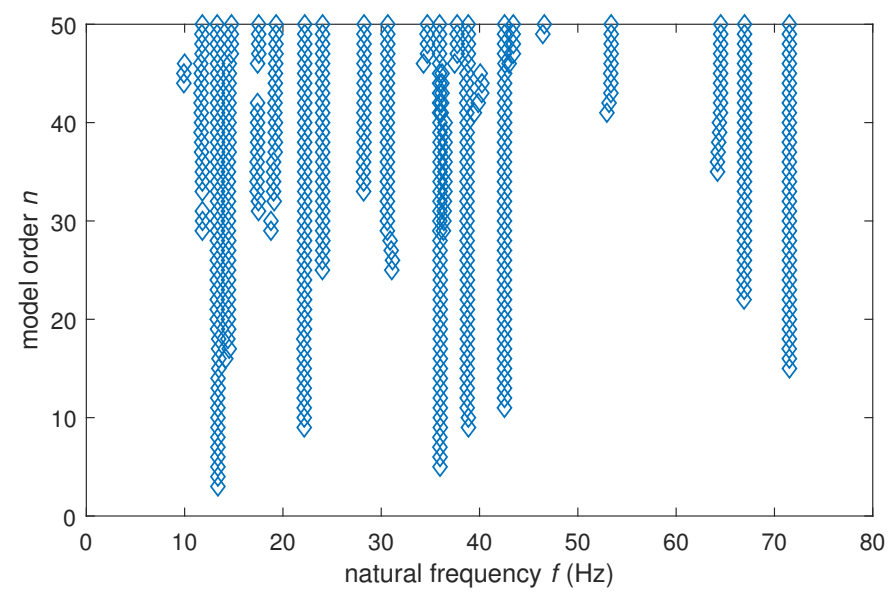

Figure 6: Stabilization diagram with Test 1.
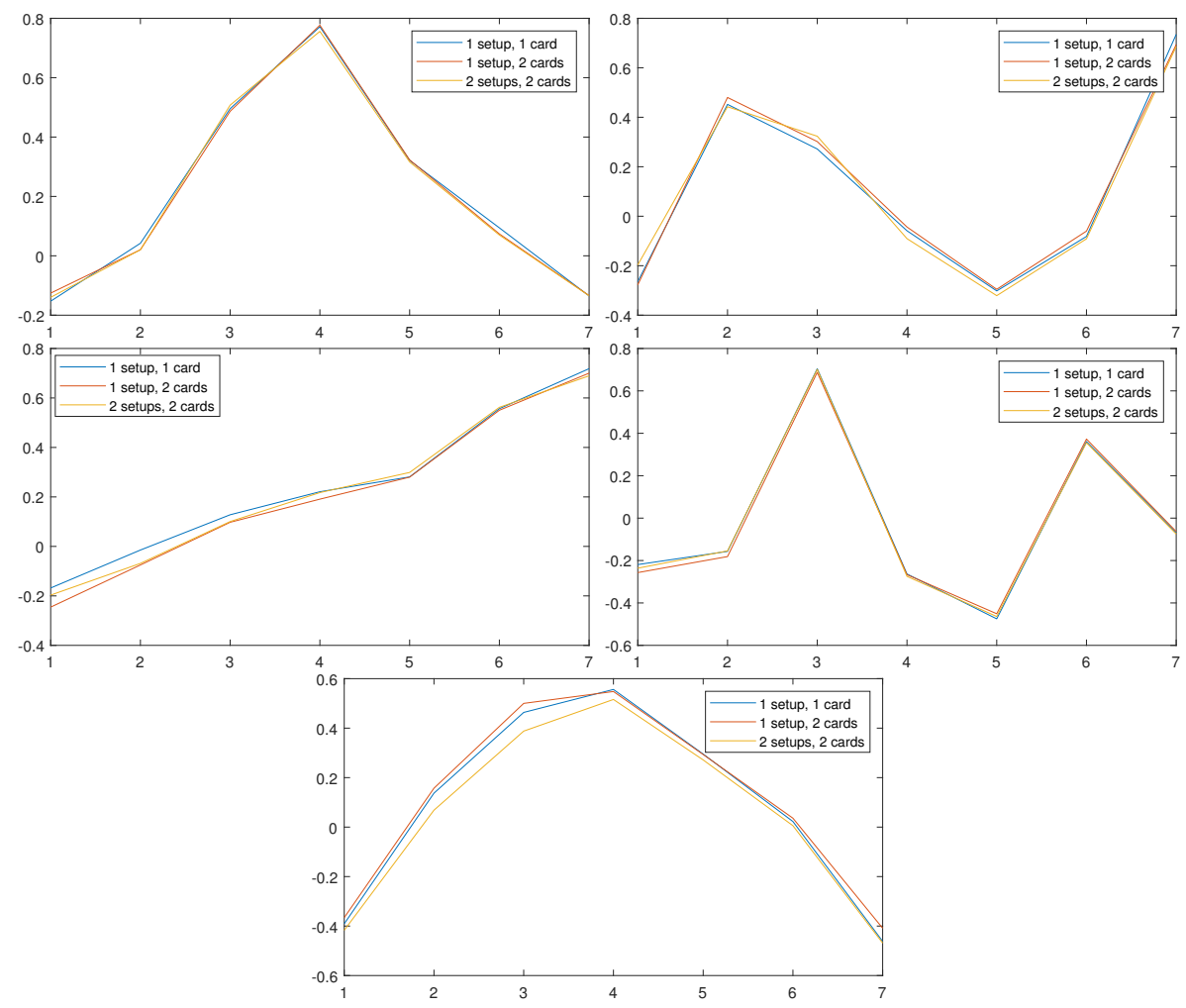

Figure 7: Mode shapes obtained with the three tests. 
TABLE I: IDENTIFIED FREQUENCIES $f$ [Hz] AND DAMPING RATIOS $\xi$ [\%].

\begin{tabular}{l|lrrrrr}
\hline & & Mode 1 & Mode 2 & Mode 3 & Mode 4 & Mode 5 \\
\hline Test 1 & $f$ & 13.31 & 22.22 & 35.93 & 42.55 & 71.52 \\
& $\xi$ & 3.64 & 2.85 & 0.77 & 1.08 & 0.29 \\
Test 2 & $f$ & 13.73 & 22.09 & 35.94 & 42.43 & 71.48 \\
& $\xi$ & 7.70 & 2.50 & 0.92 & 1.20 & 0.37 \\
Test 3 & $f$ & 13.71 & 22.10 & 35.78 & 42.54 & 71.47 \\
& $\xi$ & 8.42 & 2.81 & 0.75 & 1.38 & 0.43 \\
\hline
\end{tabular}

\section{CONCLUDING REMARKS}

With the proposed data recording and synchronization strategy, it is possible to obtain vibration measurements of complex structures at many locations with only two wireless sensor platforms and few sensors in a simple way. The subspace-based merging strategy for multiple setups allows an efficient modal analysis with just one system identification step, where no modal tracking is required between the setups, offering a theoretically sound approach. A possible development for future works is the direct synchronization of the acquisition with the "SYNC" input of the ADC. This input "SYNC" allows on rising edge to make an acquisition at a precise moment. The FPGA of each card can thus synchronize this input "SYNC" on a divider of the PPS (pulse per second generated by the GPS) and thus achieve the acquisition at the same time. With this method, the interpolation step can be avoided, saving computation time and thus energy consumption.

\section{REFERENCES}

1. Farrar, C. and K. Worden. 2007. "An introduction to structural health monitoring," Philosophical Transactions of the Royal Society A: Mathematical, Physical and Engineering Sciences, 365(1851):303-315.

2. Le Cam, V., M. Döhler, M. Le Pen, I. Guéguen, and L. Mevel. 2016. "Embedded subspacebased modal analysis and uncertainty quantification on wireless sensor platform PEGASE," in Proc. 8th European Workshop on Structural Health Monitoring, Bilbao, Spain.

3. Cho, S., C.-B. Yun, J. P. Lynch, A. T. Zimmerman, B. F. Spencer Jr, and T. Nagayama. 2008. "Smart wireless sensor technology for structural health monitoring of civil structures," Steel Structures, 8(4):267-275.

4. Döhler, M. and L. Mevel. 2012. "Modular subspace-based system identification from multisetup measurements," IEEE Transactions on Automatic Control, 57(11):2951-2956.

5. Döhler, M., X.-B. Lam, and L. Mevel. 2013. "Uncertainty quantification for modal parameters from stochastic subspace identification on multi-setup measurements," Mechanical Systems and Signal Processing, 36(2):562-581.

6. Le Cam, V., A. Bouche, and D. Pallier. 2017. "Wireless Sensors Synchronization : an accurate and deterministic GPS-based algorithm," in Proc. 11th International Workshop on Structural Health Monitoring, Stanford, CA, USA.

7. Peeters, B. and G. De Roeck. 1999. "Reference-based stochastic subspace identification for output-only modal analysis," Mechanical Systems and Signal Processing, 13(6):855-878.

8. Döhler, M. and L. Mevel. 2012. "Fast multi-order computation of system matrices in subspace-based system identification," Control Engineering Practice, 20(9):882-894. 